\title{
Post-traumatic stress disorder after intensive care
}

This patient recounts her experiences of post-traumatic stress disorder after she received supportive treatment in intensive care

\author{
Sarah Wake patient ${ }^{1}$, Deborah Kitchiner cognitive behavioural therapist ${ }^{2}$ \\ 'University Hospital Wales, Cardiff CF14 4XW, UK; ${ }^{2} \mathrm{CBT}$ Cardiff, Cardiff, UK
}

This is one of a series of occasional articles by patients about their experiences that offer lessons to doctors. The BMJ welcomes contributions to the series. Please contact Peter Lapsley (plapsley@ bmj.com) for guidance.

Endless days and nights filled with strange broken sleep. A sea of fragmented menacing faces and shadows swimming through erratic beeps and bells. A large cackling face floating over me, constantly morphing and changing shape. The staring old lady in the bed opposite, her sallow skin disintegrating, eyeballs disappearing to reveal deep dark holes from which cockroaches crawled. Her weary face melting like wax into a big grey smudge. Deafening, haunting laughter filling every space. Blood seeping through holes and cracks in my skin, forming a puddle of red around me. Small insects scuttling up my arms and legs. My chest locked to the bed with wires and straps, as a plastic mask repeatedly smothered me. A strangling sensation around my neck. A warm metallic taste. An invisible force pinning my body down as a dark curtain was drawn closed.

These are my memories of intensive care. They formed the fabric of reality that I would take forward and recall vividly in my dreams for many months afterwards. Such fragmented delusional memories made it extremely difficult to understand and make sense of what really happened to me. This prevented my psychological recovery and led to the development of post-traumatic stress disorder (PTSD).

\section{A surreal experience}

In July 2011 I was coming towards the end of my foundation training, fit and excited about an upcoming month off and a trip to trek Mount Kilimanjaro. My day started as any other, struggling through a ward round, but ended as a patient intubated in intensive care. I had had an asthma attack, which was treated aggressively with salbutamol. Unfortunately my body reacted to the large dosage and I was pushed into severe metabolic acidosis, with exhaustion, cardiac arrhythmia, and several biochemical imbalances as a result. I required supportive treatment in intensive care for many days.
While on the intensive care unit a combination of my illness, unnatural environment, sedatives, and opioids caused me to experience visual and tactile hallucinations and to feel extreme anxiety and confusion. I was repeatedly prescribed sedatives when I had episodes of chest pain, altered limb sensation, panic, and breathlessness. I now understand that a lot of these symptoms were caused by extremely high lactate levels, yet at the time I was offered no explanation and made to feel an anxious nuisance who could be silenced with a benzodiazepine. Ironically this would only increase my future delirium and confusion. My stay in the unit was prolonged while awaiting the availability of a lower dependency bed. As a result I became increasingly aware of my dynamic environment and formed vivid memories of surrounding sights, smells, and sounds.

\section{Not only war veterans}

PTSD has traditionally been associated with traumatic stressors such as combat, violent assault, and survival of natural disasters. Recently there has been increased awareness of symptoms occurring after critical illness and injury. Following a stay in intensive care, 1 in 10 patients develop symptoms of PTSD. It is one of the most common and most distressing, yet least talked about, problems associated with intensive care medicine. I had no warning about this association on my discharge. As a doctor I felt I was expected to be strong so initially hid my feelings of vulnerability and anxiety. I avoided speaking about what had happened. I didn't want to get upset, upset anyone else, or return to the uncomfortable memories.

\section{Return to darkness}

Unbeknown to me at the time, the symptoms of PTSD can commonly take weeks to months to manifest. By this time I had started a rotation in a tertiary neonatal intensive care unit, with constant reminders of the intensive care environment. Gradually the sights, noises, and smells overwhelmed me. Flashbacks became increasingly intrusive. At night I dreaded going to sleep, 
as I would have vivid dreams where I was being intubated while fully conscious but unable to communicate. I experienced repeated sensations of suffocation and would awake coughing, breathless, and drenched in sweat. Usually a placid person, I began to feel very anxious, irritable, and easily startled. I couldn't concentrate for any length of time and found myself "blanking off" frequently. After finishing a particularly busy shift, my chest became tight, my heart raced, and I became breathless. Feeling completely helpless and almost hysterical I knew I could not return to the hospital.

One of the key symptoms of PTSD is avoidance of any reminders of the stressor, and I took this to the extreme. I would make detours while travelling to avoid the sight of a hospital. I could not watch hospital documentaries or dramas and hid all my medical textbooks and journals. I enjoyed sport but began to avoid exercise, as I was terrified of the sensation of breathlessness. Even certain inanimate objects filled me with fear. I still cannot bear a shower curtain to be drawn as it reminds me of closed hospital curtains and hidden death.

\section{Finding answers}

People with PTSD usually recognise all too painfully that there is something drastically wrong, but are often unable to put a name to their feelings or symptoms. Worryingly a recent survey by Combat Stress (a charity working with veterans of the British armed forces helping to effectively treat and support their psychological wounds, www.combatstress.org.uk) showed that fewer than half of general practitioners knew the official guidelines for diagnosing PTSD as set out by the then National Institute for Health and Clinical Excellence.

I visited my doctor several times, yet failed to receive a diagnosis. I was told I was being overly anxious and needed to relax. Each time I was met with a blank face, I felt increasingly isolated and lost.

In desperation I researched my symptoms and came across articles discussing the association between intensive care units and PTSD. With overwhelming relief I read similar patients' experiences. I started a lengthy course of private psychotherapy involving exposure and eye movement desensitisation and reprocessing. I was initially cynical about this method, but it played a large part in my recovery. Gradually the distressing memories became less vivid, losing their intensity to become more organised ordinary memories. The thought of returning to a hospital environment became less uncomfortable. Using graded exposure I steadily progressed from nervously sitting in the hospital coffee shop to comfortably sitting in the intensive care to which I had been admitted.

\section{Moving forward}

Initially I wanted to resign from a career in medicine as I felt I would never be able to continue in my previous role, and had lost a lot of trust in the profession. However after four months of treatment I started to gradually return to work and successfully resumed full time work eight months after I had fallen ill. This was a massive step for me. Unfortunately I have discovered that certain events can still trigger flashbacks and lead to a depression that totally immobilises me. I am unable to work around ventilation equipment for long periods. As a result I cannot complete my paediatric training, although I am determined to continue my medical career in another specialty. I know this difficult experience will increase my understanding and empathy as a practising medical professional. I hope this article will bring awareness of the prevalence of anxiety, depression, and PTSD in patients after critical illness.

Competing interests: All authors have completed the ICMJE uniform disclosure form at www.icmje.org/coi_disclosure.pdf (available on request from the corresponding author) and declare: no support from any organisation for the submitted work; no financial relationships with any organisations that might have an interest in the submitted work in the previous three years, no other relationships or activities that could appear to have influenced the submitted work.

Provenance and peer review: Not commissioned; not externally peer reviewed.

Accepted: 4 January 2013

Cite this as: BMJ 2013;346:f3232

๑ B BMJ Publishing Group Ltd 2013 


\section{What I have learnt from this experience}

- Mental health problems can affect anyone, regardless of status and circumstance. Unfortunately they remain poorly understood by many health professionals

- The medical profession is poor at accepting and understanding colleagues with mental health problems, which I found highly frustrating PTSD is a normal reaction, in normal people, to terrifying experiences. Despite this I often felt I was being weak and ridiculous owing to the reaction of those around me

- The psychological aftercare of patients is currently neglected in the United Kingdom, with less than a third of hospitals offering support on discharge. The invisible psychological barriers patients need to overcome to return to their previous functioning can be more disabling and harmful than the initial period of physical illness

- General practitioners in particular need to be educated about PTSD, as it is to them that affected people are most likely to present

- The current treatment available on the National Health Service for patients with PTSD is poor, with few specialist centres and substantial waiting lists in excess of months. I was lucky to have financial help for excellent private treatment, yet for many this is not an option

- Becoming an inpatient is a belittling experience. It is scary, lonely, and you feel helpless

- Even if a patient has medical knowledge, it should not be assumed they understand the process occurring to them. I was often left without an explanation for the procedures and treatments I received and felt nursing staff thought I would rather be left alone. Frequent reassurance, explanation, and interaction with patients are vital in reducing the anxiety and fear associated with intensive care

- Some of the associations increasing the risk of PTSD after a stay on the intensive care unit are modifiable. Medical professionals should be aware of the psychological side effects of certain drugs (in particular benzodiazepines) and avoid their use if possible

\section{A clinician's perspective}

It has long been recognised that people can develop psychological symptoms after their involvement in a major traumatic event. ICD-10 (international classification of diseases, 10th revision) classifies the trauma as an event "of an exceptionally threatening or catastrophic nature, which is likely to cause pervasive distress in almost anyone." This distressing reaction is more clearly defined in the Diagnostic and Statistical Manual of Mental Disorders, fourth edition, criteria of a response of fear, horror, or helplessness. In this case, Sarah experienced an asthma attack that led to intubation and treatment in intensive care. She described thinking she was going to die and feeling extremely helpless.

PTSD symptoms include three subtypes; re-experiencing phenomena such as intrusive thoughts or recurrent dreams; avoidance and numbing; and increased arousal-for example, irritability or hypervigilance.

I treated Sarah for several months as an outpatient for PTSD. I used a trauma focused psychological therapy, eye movement desensitisation and reprocessing (EMDR) to process her traumatic memories, and graded exposure to help Sarah back to hospital environments and eventually back to work.

EMDR is one of two NICE recommended psychotherapies for PTSD. EMDR therapy shows that the mind can heal itself from psychological trauma much as the body recovers from physical trauma. EMDR is centered on the theory that bilateral stimulation allows traumatic memories to be processed. Bilateral stimulation is primarily eye movements but can be other types of stimulation such as hand taps or sounds. During EMDR the patient holds the worst image of the trauma in mind with the associated negative sensations and cognitions. The client then tracks the therapist's hand as it moves back and forth across the client's field of vision. Internal associations then arise and the client begins to process the memory and disturbing feelings. In successful EMDR therapy, the meaning of painful events is transformed on an emotional level.

During therapy Sarah's memories were very jumbled up and she was often unsure as to what was real. Sedatives, such as benzodiazepines, can create disorientation and confusion, which can be extremely frightening for patients. Sarah regularly spoke of not understanding what was happening to her while on intensive care. She frequently described how nobody had explained things to her and she constantly felt confused and frightened. She was unable to communicate this at the time owing to sedation or intubation. If medical or nursing staff had explained what they were doing and what was happening every time they were with Sarah, even if she was unconscious, then this could have positively affected her experience.

PTSD after admission to intensive care is common and it is important that before they leave hospital patients are given information describing symptoms they may experience and where and when to seek help. Early intervention for PTSD can lead to better treatment outcomes.

Finally, support and understanding from medical colleagues to their peers is vital when someone experiences PTSD as Sarah did. Medical staff can feel that they should be immune to this kind of mental health problem. These sorts of attitudes could lead to feelings of shame and embarrassment, which can obstruct recovery.

Deborah Kitchiner, cognitive behavioural therapist 